\title{
Assessment of stratification of the breeds of the underground cavity roof
}

\author{
Tamara Makeeva ${ }^{1, *}$ and Vitaly Trofimov ${ }^{2}$ \\ ${ }^{1}$ Moscow State University of Civil Engineering, 129337, 26, Yaroslavskoye Shosse, Moscow, Russia \\ ${ }^{2}$ Russian Academy of Sciences, Institute of problems of complex development of bowels, 111020, 4, \\ Kryukovsky Tupik, Moscow, Russia
}

\begin{abstract}
Authors of the research consider the approach to the assessment of possibility of formation of stratification and shift cracks in the roof of extended horizontal cavity, based on the method of boundary elements. The algorithm implements the iterative procedure of variation of the formed crack length with control of tension in its tip. At the same time, the angle of the crack coasts closing is defined by the tensile strength of the layer material. The crack with smooth closing of coasts is formed in case of equality to zero this durability. The mechanism of failure forming over the developed space with vertical pipe type walls is considered. The results received by the authors, can be useful as the offered approach allows to model the destructions localization in the massif of rocks, location and sizes of the arising cracks of stretching and shift if there are strength defects in the massif.
\end{abstract}

\section{Introduction}

Situations of forming of cameras, which are large in the area and extent and freely hanging roof quite often occur in the practice of construction of underground structures. Such mining operations is characteristic of rocky breeds when the area of the hanging roof can reach thousands of square meters without stability loss. The same situation is characteristic of the mining industry when, clearing works carried out during working off of coal layers, can develop for hundreds of meters without destruction of the main roof.

It seems to be undoubted, that the state and behaviour of the roof of such cavity is defined by its geological structure first of all. The variety of options of such structure generates a set of approaches and methods for the evaluation of its state and possible destructions. Further we will consider the use of numerical algorithms as the most universal and effective for the tasks of geomechanics, which include stability of the roof of the developed space.

The horizontal development $2 \mathrm{~L}$ long and $\mathrm{h}$ height, located at $\mathrm{H}$ depth from day surface is considered.

At the same time, the authors consider the situation, when the mining operations are located in the massif of the layered structure, which is characteristic of sedimentary rocks. Such massifs have the anisotropic distribution of deformation and strength properties

* Corresponding author: makeeva13new@yandex.ru 
caused by availability of numerous layers of rocks of various power and material structure. It is noted that the behaviour of such massif during the mining operations is generally defined by deformation of the layer, which is often the only one and has rather huge power and rigidity (the main roof). Deformation of this layer defines the behaviour of the land surface, load for pillars, gas and water occurrence substantially $[1,2,3]$.

At the same time all the overlying layers carry out the role of passive load of this roof. At the same time, the layers located under the main roof (direct and false roofs) are as a rule rather dismembered by cracks and they are of little importance during the deformation of the massif. So, they can be neglected during the creation of the settlement scheme of the massif.

Numerous works were performed within the creation of various models of the layered roof deformation [4-7], with the use of numerical models [8, 9] which were used for the determination of the law of distribution of dynamic soil cracks under the influence of overlying bedded structures $[10,11]$. That should be noted that the considerable research is connected with the use of the frame models [12-15], based on the use of beam-and-hinge structures. It is possible to note the approach to the analysis of the block roof on the basis of the method of discrete elements with the defining Mohr-Coulomb function [16-20].

The main roof may contain thin low-strong layers which have certain durability on stretching and shift, and under certain conditions they can lead to the main roof stratification.

Further we will consider for simplicity, that there is only one such layers in the main roof, which separates a part of the main roof, which power is $h l$ (fig. 1). At the same time the durability of the contact $f$ this layer with the overlying breeds is insignificant; and that promotes the rupture of this contact and the corresponding stratification of breeds with the formation of a gaping crack.

The difference in the deformation parameters of the main roof layers, is usually insignificant, and it can also be neglected. The authors consider that the massif of rocks is described with the linearly-and-elastic, uniform and isotropic model with the following parameters: E - the module of elasticity, $v$ - Poisson's coefficient of rocks, $\gamma$ - average specific weight.

We arrange the beginning of the rectangular Cartesian coordinate system in the middle of the development, we will direct the $0 x$ axis on its roof, $0 y$ axis vertically up, and $0 z$ axis parallel to face. Besides, we consider that the development extent in the direction of consider $\mathrm{z}$ axis is rather big to assume the accomplishment of the conditions of flat deformation near 0xy surface at least.

The task consists in the determination of conditions of the emergence of such a stratification as well as in the creation of numerical algorithm of the description of the deformation processes proceeding at the same time and connected with the formation and progress of a gap and shift crack. The task becomes simpler, because of the fact that the trajectory of the crack progress is known in this situation, as it is caused by natural lamination of the roof breeds. In case of the accomplishment of its emergence conditions, it will extend parallel to the development roof at $h l$ distance from it. We will use the option of the method of boundary elements (method of explosive shifts) [21-23] for the solution of this task.

At the same time the extended clearing development and the line of contact of the breeds in its roof are modelled by two corresponding sets of boundary elements (to $\mathrm{M}$ and $\mathrm{N}$ items accordingly). The deformation properties of the elements, which are modeling the development and contact differ considerably as the splash of normal shifts for the elements of the last has to be always such, that their banks are close, or moved apart with the formation of the gaping stretching crack. The continuous control over the implementation of this condition is exercised during the task solution. The banks for all the elements are 
close initially, i.e. the gaping cracks are absent. There can be some conditions in case of change of the intense deformed condition of the settlement area, when the banks of the elements disperse and the gaping crack is formed.

The normal shifts of the banks, when there is formally their mutual penetration each other are allowed for the development elements. The geo-mechanical interpretation of this situation consists in that the excavation has some height, so the shift of the upper bound of the element can be explained by the roof sedimentation, and the lower bound can be explained by the soil raising. Thereby, the mutual penetration of the banks just corresponds to the reduction of the development gleam.

As for the sizes of tangent splash of shifts, they can have any sign, both for the development elements, and for the contact.

\section{Statement of the Issue}

So, we consider the situation when a clearing development with a flight of the hanging roof $2 \mathrm{~L}$ was formed during working off of a horizontal formation. At the same time there is a layer with power $h l$ in the main roof put by elastic breeds with parameters $E$ and $v$, adjoining the developed space directly and connected with the other roof rocks with thin low-strength layers. At the same time, it is initially unknown whether there was a gap at the contact and what its length, if it has occurred. It is also represented in figure 1 schematically in a general view, where $\ln$ is the length of a possible gap crack.

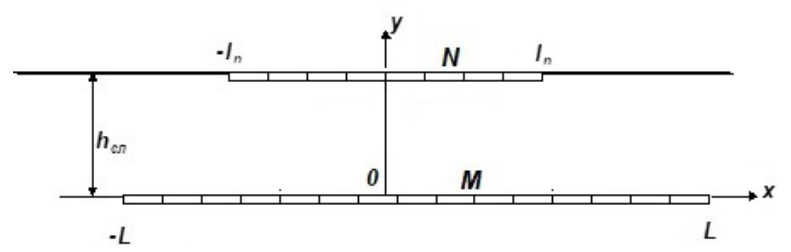

Fig. 1. Diagrammatic representation of the design area.

Boundary conditions on the surfaces of the development and contact, including the possible crack, are set by the parameters $\sigma_{n}, \tau_{n}, D_{n}, D_{t}$ (normal and tangent tension on the crack as well as normal and tangent splash of shift of the crack banks). At the same time, it should be kept in mind that according to the determination $D_{n}$ and $D_{t}$ are equal the differences of the corresponding absolute shifts of the lower and upper banks of each boundary element, taking into account the set direction of traversal.

The following conditions should be satisfied throughout the whole development, i.e. for all its boundary elements, in case of lag of the roof:

$$
\sigma_{n}=\tau_{n}=0
$$

If there is roof caving on the soil, the following condition should be for the boundary elements on the site of contact:

$$
D_{n}=h,
$$

and on sites of lag the condition is still (1).

Besides, it is possible to consider that in the places of roof caving during the development of a mined-out space Dt has the same values which it had at the time of contact of the roof and the soil.

Now we consider the boundary conditions for the elements, modeling the contact of breeds in the development roof. At the same time, we can distinguish three characteristic 
zones adjoining to each other, according the form of interactions of the contact banks. Namely:

1) zone of the gaping crack (if $x<\ln$ ), if it was formed. The conditions for it come down to the lack of tension on the bank, just like as well for the development

$$
\sigma_{n}=\tau_{n}=0
$$

In this case, volumes of splashes of shifts are naturally nonzero, $D_{n} \leq 0$ and $D_{t} \neq 0$. At the same time the first inequality is connected with the banks divergence of some elements on the line of contact and characterizes them as the revealed crack.

It is necessary to distinguish two more adjacent zones on the rest of contact:

1) throughout the first zone, adjoining the gaping crack, i.e. from $x=l_{n}$ to $x=l_{t}$, conditions are met $D_{n}=0$ и $D_{t} \neq 0$, i.e. there is no banks divergence there, and the shift is present still;

2) if $x>l_{t}$ the conditions $D_{n}=0$ and $D_{t}=0$ are fulfilled, i.e. there are no banks divergence and shift.

Thus, in fact two cracks can be created at the contact one of them is a gap crack $l_{n}$ long, the other one is shift crack $l_{t}$ long.

It is obvious that the values of parameters $l_{n}$ and $l_{t}$ are unknown in advance and have to be received from the task solution, proceeding from these or those criteria of destruction using the strength properties of the contact which can be very non-uniform on its length. In this regard it is possible to set these two parameters randomly within numerical modeling (if $l_{n}<l_{t}$ ), the parameters determine the mentioned zones, and to receive the corresponding tension distributions and shifts as a result.

However, let us mention the following circumstances connected with strength characteristics of the contact of the considered $h l$ layer with as it was mentioned, it is rather small, it is equal to zero in the limit. At the same time, the shift strength $\tau_{p}$. can be any. However, $\sigma_{p}$ and $\tau_{p}$ there have to be less than the corresponding volumes for the layer material in order to guarantee the possible destruction at the contact, but not in the layer.

\section{Results and Discussion}

As we could expect, the calculations prove that for a small length of the crack $l_{n}$, i.e. if $l_{n}$ is less than $l_{n}^{\kappa}$, which is defined by tensile strength, at its continuation in close proximity to the tip there is a concentration of the stretching normal tension $\sigma_{n}^{l}$, which can exceed $\sigma_{p}$ considerably. Therefore, the chosen $l_{n}$ does not correspond to the conditions of loading in those boundary elements where $\sigma_{n}^{l}>\sigma_{p}$, in fact the stratification should take place and these elements should be attached to the crack with the corresponding boundary conditions. It should repeat, i.e. the size of the crack needs to be increased until $\sigma_{n}^{l}<\sigma_{p}$ in the boundary element, next to the tip.

Thus, as a result of some iterative procedure the extreme extent of the equilibrium stretching crack $l_{n}^{\kappa}$ corresponding to the length of development $L$, is defined for the set $\sigma_{p}$ and $l_{t}$.

The essence of the used algorithm can be briefly stated as follows. A set of the boundary elements modeling the development remains invariable during the task solution. At the same time the splashes of shifts received for these elements are interpreted as the shift of a roof and the soil of the development, defining their lowering and rise respectively. 
As an example, the continuous lines in figure 2 show the isolines of vertical tension $\sigma y$ in the neighborhood of horizontal mining $L=50 \mathrm{~m}$ long, located at the depth $N=500 \mathrm{~m}$ for $h_{l}=20 \mathrm{~m}$. For comparison in case of the lack of the layer in the mining roof the same tension is presented by the dashed lines.

When calculating it was accepted that the size is limited by 20 meters $\left(l_{t}=20 \mathrm{~m}\right)$, i.e. it was considered that if $x>20 \mathrm{~m}$ than $D_{n}=D_{t}=0$. In other words, if $\mathrm{x}>20 \mathrm{~m}$ rather strong contact takes place and its destruction is impossible under any circumstances (i.e. $\tau_{p}$ it is great, for our case it is $1000 \mathrm{~kg} \mathrm{per} \mathrm{cm}^{2}$ ). The check of lack of concentration tension in the stretching crack tip was carried out as the strength criterion on stretching, i.e. it was supposed that $\sigma_{p}=0$.

As for the size of the used criterion of destruction, it was determined as the result of several iterations by $1 \mathrm{n}$ which gradually increased, since $10 \mathrm{~m}$. As a result, it made up $18 \mathrm{~m}$.

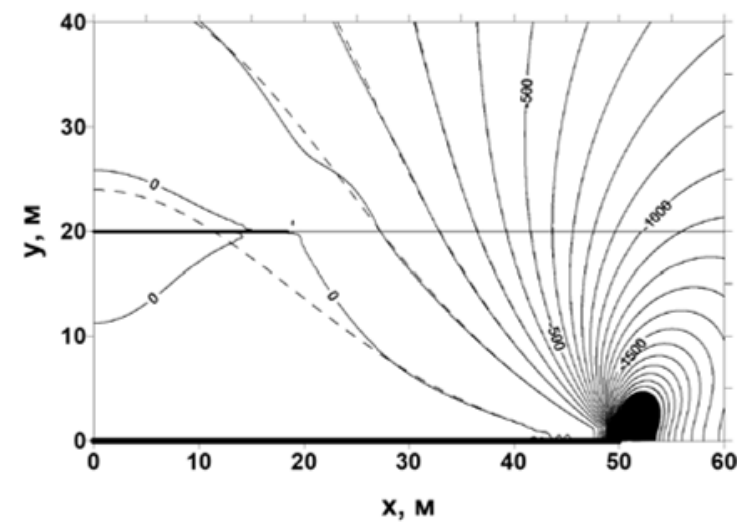

Fig. 2. Isolines of vertical tension $\sigma y$ in the neighborhood of horizontal mining $\mathrm{L}=50 \mathrm{~m}$ long located at the depth of $\mathrm{N}=500 \mathrm{~m}$ for $\mathrm{hl}=20 \mathrm{~m}$. (Dashed lines are made for the lack of a layer in the mining roof).

Total distribution of the sizes $D_{n}$ and $\sigma_{n}$ on $\mathrm{x}$ in the elements of contact is shown in figures 3,4 . At the same time there is no both positive or negative splash of normal tension $\sigma_{n}$ in the point $x=18 \mathrm{~m}$, according to the chosen criterion of durability. In this connection there is smooth interlocking of the gap crack banks.

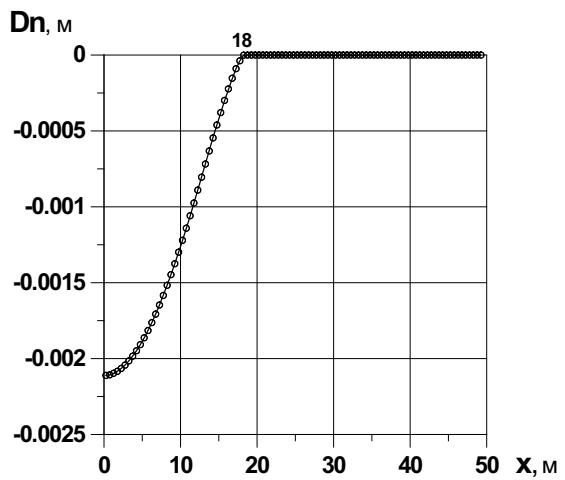

Fig. 3. Equilibrium distribution of splash of shifts $D_{n}$ along $x$ in the contact elements. 


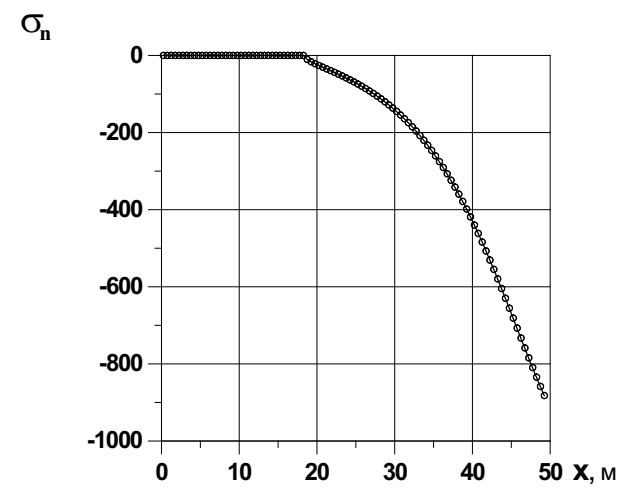

Fig. 4. Equilibrium distribution of compression tension $\sigma_{n}$ along $x$ in the contact elements.

At the same time the distribution of tangent tension $\sigma \mathrm{t}$ has a feature, reflected in figure 5 , if $x=20 \mathrm{~m}$. However, owing to the put strength restrictions, this concentration cannot lead to any germination of shift crack beyond $x=20 \mathrm{~m}$.

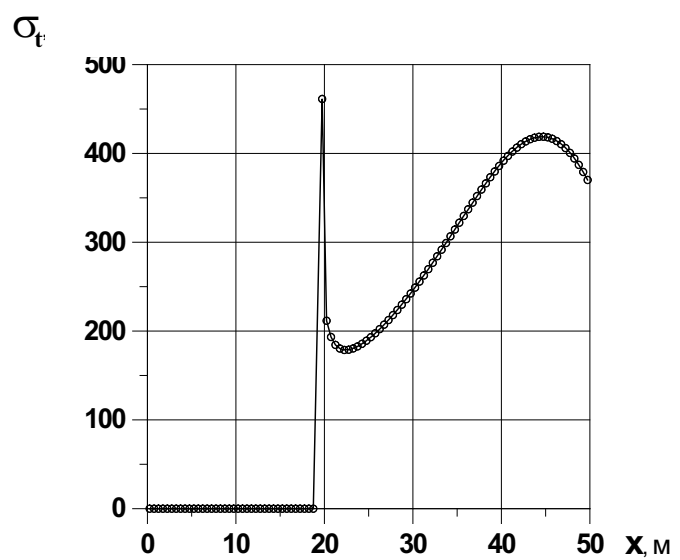

Fig. 5. Distribution of shift tension $\sigma_{t}$ along $x$ in the contact elements after establishing the separation crack balance.

Let us note the close interrelation of the sizes of cracks of stretching and shift. In case of the increase in lt there is a gradual reduction of tangent tension concentration along the shift crack tip for $\mathrm{x}=1 \mathrm{t}$ which $\rightarrow 0$ with the unlimited growth of lt. Proceeding from it, the actual length of the shift crack is defined only by the size of shear strength $\tau \mathrm{p}$. If at the same time $\tau \mathrm{p} \rightarrow 0$, then the length of $1 \mathrm{t}$ can become as much as big though it does not make any sense to consider too big lt (in our case $>100 \mathrm{~m}$ ), as the distribution of tension and shifts in the array becomes almost invariable.

As for the stretching crack, the carried-out calculations proved that the more to put the size $1 t$, the more ln will be, other things being equal. However, proceeding from the physical reasons, it cannot extend the stope surface at any lt values and any criteria of destruction to stretching.

The moment of the beginning of the stretching crack growth can be approximately defined proceeding from the sizes of the zone of the stretching vertical tension in case of the lack of layers in the mining roof. At the same time for the maximum depth dimension of this zone (at $\mathrm{x}=0$ ) we have the approximate ratio [23] 


$$
y_{\max }=L \sqrt{\frac{L}{H}} .
$$

$y_{\text {max }}=h_{c л}$, i.e. the moment when all the power of the layer is covered by the stretching zone in case of $x=0$ can be considered to be the condition of emergence of a crack. Then we will obtain the formula for $L_{b}$ :

$$
L_{b}=\sqrt[3]{h_{c \Omega}^{2} H} .
$$

The same task can be considered within the theory of cracks according to which the followig feature takes place in the tip of the stretching crack: $\sigma_{n}=\frac{K_{I}}{\sqrt{\varepsilon}}$,

where $\varepsilon$ - distance from the crack tip,

$\mathrm{KI}$ - the coefficient of tension intensity determined by the geometrical configuration of area and loading conditions.

The nature of its change during the growth of the crack defines the crack's behavior, and its size can be determined approximately according to the size $\sigma_{n}$ in the next boundary element [25]. That does the approach, described above, fair. However, sizes $\sigma_{p}$ and $K_{I}$ should be coordinated properly.

Now there is an opportunity to determine the parameters of stratification cracks and their influence on the intense deformed condition of the rock mass within the used model.

In this regard we will consider the intense deformed condition of the leaning array, however we will suppose that impact of breeds stratification on the array condition. If the mining length is $\mathrm{L}=50 \mathrm{~m}$ the equilibrium stratification crack has length $\sim 45 \mathrm{~m}$, in comparison with $\mathrm{lp}=35 \mathrm{~m}$ for $\mathrm{hl}=20$ m now.

Figure 6 demonstrates the vertical relocations of the mining crack banks (upper -1 , lower -2 ), roofs (3) and soil (4).

Sedimentation of the lower bank and the roof of the crack practically coincide, the distinction is $\sim 1 \mathrm{~cm}$. It means that the breeds of the layer are not deformed in the vertical direction and fell as a unit $(\sim 35 \mathrm{~cm}$ in the center).

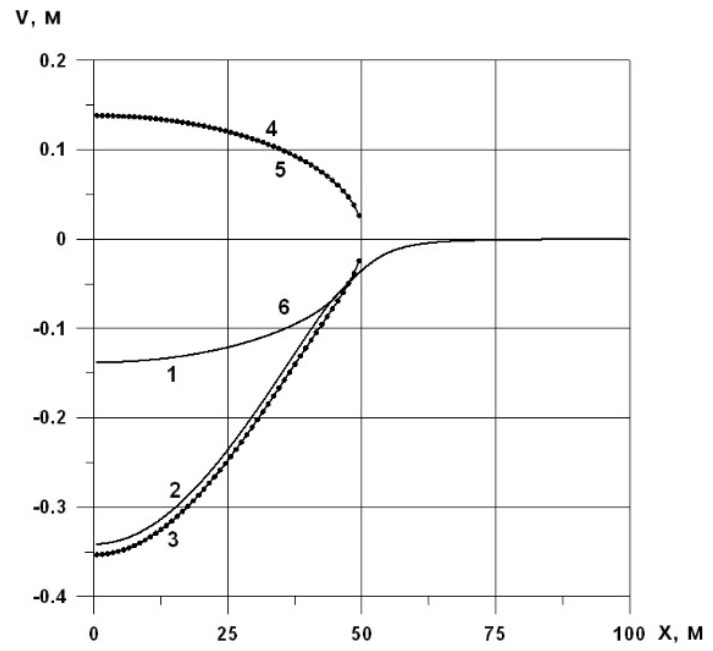

Fig. 6. Relocation of the separation crack banks and the development surfaces at the moment of the equilibrium state establishing. 
There is no vertical tension in this layer (or they are close to zero) and, therefore, there are no conditions for its further stratification. The points show the shifts of the soil (5) and the roof (6) in case, when there is no stratification. It is obvious that stratification does not influence on the raising of the soil of the development and therefore (4) and (5) coincide practically. We should note the feature consisting in the fact that the sedimentation of the upper bank of the crack of stratification (1) coincides with the development roof subsidence (6) without a stratification crack.

The interpretation of slashes is slightly more difficult for the set of the crack elements. The solution begins with the randomly set length of a crack $l_{p}$, which can appear to be lessor more than the length which really should be in the considered geo-mechanical situation. This length is quite defined by the set task parameters. The essence of the applied algorithm consists in the iterative adjustment of the set length by addition or decrease of the boundary elements which are a part of the crack. Tension of the extreme boundary element among the crack elements is the criterion for carrying out such a measure. As the crack banks in the equilibrium state should meet each other smoothly, the slash of shifts should be equal zero in it, or tension, normal to the element plane, is zero too. If tension is more than zero in this element as the calculation result, i.e. tension is stretching, then the crack is too long and it is necessary to remove at least one element, otherwise to add an element. Varying the quantity of elements, we can achieve normal tension which would be approximately zero. Thereby, the required equilibrium length of the stratification crack will be defined.

The algorithm essence consists in the fact that the development of the shift crack is not considered.

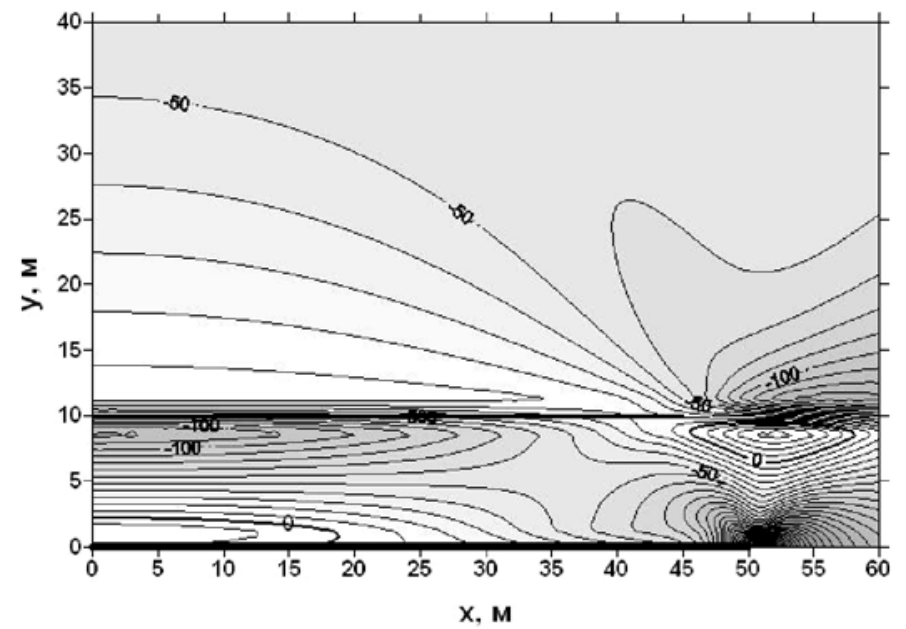

Fig. 7. Isolines of horizontal tension $\sigma_{x}$ during the establishment of an equilibrium state.

That can proceed until the collapsed and loosened breed close the whole gleam and prop up the newly-formed roof. It can be the mechanism of the shrinkage formation of the rock mass in the form of "pipe" over the mined-out space with almost vertical walls, which is quite often observed in practice.

Let us look at Figure 7, which presents the horizontal tension isolines $\sigma x$. Let us note the existence of pulling stresses in the central part of the roof and over the face, despite the squeezing initial tension $\sigma_{x}^{0}$ which is equal to the coefficient of a side thrust. In case of the lack of a stratification crack $\sigma \mathrm{x}<0$, i.e. the compression is $[24,26]$. 
It is obvious that the aforesaid has no absolute character, and should be correlated to the concrete parameters, describing a mining situation: $\mathrm{H}, \mathrm{L}, \gamma, \mathrm{KIC}, \mathrm{l}_{\mathrm{ip}}, \mathrm{E}, \mathrm{v}, \mathrm{m}$.

\section{Conclusion}

The considered approach allows to model the localization of destructions in the array of rocks, location and sizes of the arising tensile and shear cracks in case of the presence of strength defects in the array.

\section{References}

1. C. Fan, S. Li, D. Elsworth, J. Han, Z. Yang, Energy Science \& Engineering 8(4), 1015-1028 (2020)

2. Z. Q. Song and J. Hao, Journal of Shandong University of Science and Technology (Natural Science) 38(1), 1-15 (2018)

3. D.F. Yun, Z. Liu, W. D. Cheng, Z. D. Fan, D. F. Wang, Y. H. Zhang, International Journal of Mining Science and Technology 27(1), 179-184 (2016)

4. H. Yan, J.-X. Zhang, L.-Y. Li, R.-M. Feng, T.-T. Li, Journal of Central South University 25(2), 448-460 (2018)

5. L. Fei, Z. Jiang, Advances in Civil Engineering 1368965, 13 (2018)

6. J. Zhang, Energies 12(4), 628-640 (2019)

7. S.-C. Wang, L.-M. Dou, Z.-L. Mu, J.-R. Cao, and X.-W. Li, Shock and Vibration 8469019, 14 (2019)

8. G. Si, S. Jamnikar, J. Lazar et al., International Journal of Coal Geology 144-145, 98$110(2015)$

9. D.W. Zhou, K. Wu, Z.H. Bai et al., Bulletin of Engineering Geology and the Environment 78(2), 1025-1044 (2017)

10. W.L. Yao, Z. Yang, X.Y. Guo, Energy Sources, Part A: Recovery, Utilization, and Environmental Effects 41(3), 269-279 (2018)

11. P. Li, X. Wang, W. Cao, D. Zhang, D. Qin, H. Wang, Energies 11(1), 102-113 (2018)

12. J. Li, Y. Huang, J. Zhang, M. Li, M. Qiao, F. Wang, Advances in Civil Engineering 5929635, 11 (2019)

13. W. Wang, Y.-P. Cheng, H.-F. Wang et al., Engineering Failure Analysis 54, 150-162 (2015)

14. J. Guo, G. Feng, P. Wang, T. Qi, X. Zhang, and Y. Yan, Energies 11(5), 1041-1060 (2020)

15. Z. Li, J. Xu, J. Ju, W. Zhu, J. Xu, International Journal of Rock Mechanics and Mining Sciences 108, 67-79 (2018)

16. S. He, Y. Li, A. Aydin, Tunnelling and Underground Space Technology 72, 242-249 (2018)

17. D. Ren, D. Zhou, D. Liu, F. Dong, S. Ma, and H. Huang, Journal of Petroleum Science and Engineering 178, 497-505 (2019)

18. N. Zhang, W. Liu, Y. Zhang, P.F. Shan, X.L. Shi, Energies 13(7), 1-22 (2020)

19. P. Shan, X. Lai, Engineering Computations 37(6), 2177-2192 (2020) 
20. S. Zhang, D. Zhang, H. Wang, S. Liang, Journal of Geophysics and Engineering 15(6), 2642-2657 (2018)

21. S. Crouch, A. Starfield, Methods of boundary elements in solid body mechanics (Moscow, World, 1983)

22. S.V. Kuznetsov, V.A. Trofimov, Journal of Mining Science 43(4), 341-360 (2007)

23. S.V. Kuznetsov, V.A. Trofimov, Geodynamics and tension of Earth subsoil (Novosibirsk, 2009)

24. K.N. Trubetskoi, M.A. Iofis, S.V. Kuznetsov, V.A. Trofimov, Journal of Mining Science 35(3), 209-215 (1999)

25. S.V. Kuznetsov, V.A. Trofimov, Proceedings of the International scientific and technical conference, "The II Erzhanovsky readings" (Aktobe, 2007)

26. S.V. Kuznetsov, FTPRPI 6 (1988) 